\title{
Autostereoscopic Displays for Visualization of Urban Environments
}

\author{
Vladimir B. Markov ${ }^{\mathrm{a}}$, Stephen A. Kupiec ${ }^{\mathrm{a}}$, Avideh Zakhor ${ }^{\mathrm{b}}$, Darrel Hooper ${ }^{\mathrm{c}}$, and Gurdial S. Sainic \\ ${ }^{a}$ MetroLaser Inc., 2572 White Road, Irvine, CA 92614 \\ ${ }^{\mathrm{b}}$ University of California, Berkley, 507 Cory Hall, Berkeley, CA 94720 \\ ${ }^{c}$ Air Force Research Laboratory, Wright Patterson AFB, OH, 45433, USA
}

\begin{abstract}
Two approaches in designing autostereoscopic displays capable of providing collaborative viewing of real time 3D scenery will be presented and discussed. Both techniques provide multiscopic "look around" capabilities and are applicable for situation rooms or mobile command centers. In particular, we discuss a prospective use of these displays for interactive visualization of detailed three-dimensional models of urban areas, and the specific demands associated with managing and rendering large volumes of highly detailed information. Latest advances in scanning, survey and registration in urban areas have provided a wealth of detailed three-dimensional data and imagery. Recent events have shown a severe need and demand for systems capable in a high-level 3D visualization upon homeland security posed by terrorist actions and natural disasters within urban areas, as well as for military operations in urban terrain (MOUT). The capacity to visualize sightlines, airflow, flooding, and traffic in real time 3D within dense urban environments is increasingly critical for military and civilian authorities, as well as urban planners and city managers. Development of a high-quality 3D imaging systems is critical also for such areas as medical data imaging, gaming industry, mechanical design and rapid prototyping.
\end{abstract}

Keywords: 3D Displays, Autostereoscopic, Multiscopic, Urban Visualization, Rendering, Homeland Security

\section{INTRODUCTION}

A significant proportion of the Earth's population lives in dense urban areas, which has led to an increasing emphasis on the need to visualize the complex structure of such environments. Natural disasters, such as the Hurricane Katrina, the Southeast Asian tsunami and their aftermath, military operations, and homeland security have all demonstrated the increasing need for decision making tools allowing civilian and military authorities to both plan and monitor operations within urban areas. In particular, three-dimensional visualizations of dense urban areas are desirable in that they not only constitute three dimensional surfaces, but also the interior volume of various facilities, such buildings, underground bunkers, tunnels, utilities and infrastructure. With the advent of such programs as Google Earth, the two dimensional visualization of three-dimensional scenes has become increasingly common, but many of the practical uses of the technology are complicated by the ambiguities of two-dimensional perspectives of three-dimensional scenes. In particular, gauging the three dimensional position of paths and points as well as dealing with multlayered translucency. As a result the development of true 3D displays allowing for collaborative viewing of urban scenes will allow for a denser yet more intuitive visualization of urban areas.

Within this paper, we present the architecture of two types of autostereoscopic displays that are well suited to the collaborative display of urban environments. We will also consider corresponding software that enables the display urban areas derived from actual data or from architectural models. Both display types[1,2] are autostereoscopic and multiscopic [3] in that they do not require glasses or goggles for viewing and simultaneously displaying multiple perspectives allowing for "look around" viewing of scenes with multiple viewers. This is achieved by exploiting the very high framerate possible with deformable mirror devices (DMDs) to implement temporal multiplexing [4]. In the second design the imaging geometry is coupled with the use of the aspect from points (AFP) approach [5,6] by applying holographic optical elements to redirect the multiplexed images. The operation is enhanced through advances in graphical processing unit (GPU) technology coupled with improvements in computer bus technology to allow for the real time generation of the necessary perspectives and the high bandwidth transfer of the corresponding information to the DMD unit. 
In addition we describe the integration of urban models based on the work of one of the authors $[8,9,10]$ within the display system. Due to the proportionally greater rendering requirements associated with the multiple perspectives it is essential to optimize rendering times. As a result it is highly desirable to incorporate a combination of level of detail (LOD) data in conjunction with scenegraph-based modeling to allow for optimal control of rendering complexity. Thus the virtual reality modeling language (VRML) representations generated by the scanning and fusion software are interpreted by a customized rendering engine to allow for generation of the appropriate oblique perspectives with optimal performance.

\section{DISPLAY HARDWARE}

The capacity to display rendered computer models in true 3D has existed for a considerable amount of time, but until recently, viewing of the imagery required special glasses or goggles. While such methods have seen wide adoption in oil and gas exploration and scientific visualization where such tools are essential, widespread use has been limited due to the cumbersome nature of the viewing equipment. With the recent advent of practical autostereoscopic displays it is possible to display stereoscopic scenes without the need for glasses or goggles. The first generation of autostereoscopic displays have primarily generated stereoscopic views suited to individual viewers with two viewing zones and limited look around capabilities. New designs allow for multiple perspectives distributed across multiple viewing zones allowing for both look around and multiple viewers. Both types of the true 3D displays we employ for urban visualization incorporate multiscopic capabilities allowing for collaborative evaluation of the imagery. The two configurations described in this paper employ temporal multiplexing as opposed to the more common spatial multiplexing (lenticular and parallax barrier) techniques. The first display utilizes conventional optics while the second also incorporates a holographic optical element to allow for more flexible redirection.

\subsection{Temporally Multiplexed Autostereoscopic Display (TMAD)}

The design of the TMAD is based on the conventional optics, and its primary components are shown in Figure 1. A high speed DMD based SLM is imaged onto a diffuser element via a relay lens forming a real image. This image is then imaged by a second two-element lens system (consisting of the front and rear projection lens assemblies), which relays the image from the diffuser to a Fresnel field lens. Interspersed between two elements of the secondary lens system at the pupil is a high speed represented by the intervening box shutter, which is synchronized with the projection engine to generate a slit corresponding to the perspective being displayed on the projection engine. This slit is imaged to the exit aperture, which corresponds to the viewing position. Each position of the slit/exit aperture has a matched image associated with it. By projecting images and operating the shutter at a rate sufficient to achieve persistence of vision all of the viewing zones remain visible at the same time, creating a multiscopic display.

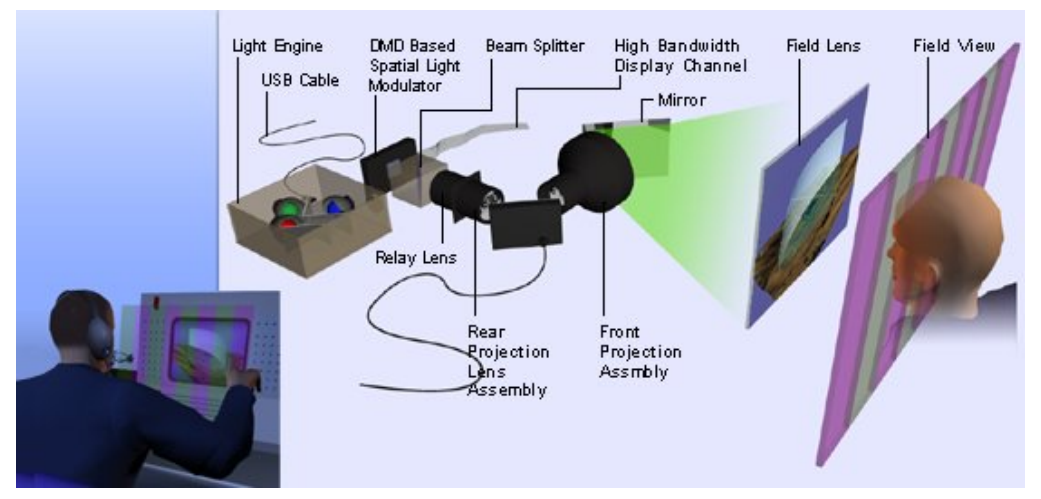

Figure 1. Temporally Multiplexed Autostereoscopic Display.

This approach has the advantage of generating a large (8-10) number of viewing zones through a single optical train wile retaining the full resolution of the projection engine. The primary challenge associated with TMAD architecture is due to an exceedingly high frame-rate required to simultaneously project the multiple images requisite for the 3D operation. This requires the use of a projection engine, which incorporates a correspondingly high frame-rate spatial light modulator, as well as the necessary rendering hardware and high bandwidth connection transfer the rendered 
frames. Advances in the raw frame-rates of DMD based SLMs coupled with advances in the speed of both modern GPUs and their associated data busses have greatly increased the viability of time multiplexed designs.

The initial prototype of the TMAD shown in Figure 2 has demonstrated up to six viewing zones in conjunction with up to four independent perspectives. The primary limitation of the initial system was the use of a conventional projector to provide imagery. As a result a maximum framerate of 85 frames per second was available, limiting the number of independent perspectives to 4 before the level of image flicker became intolerable. The prototype system has been demonstrated in conjunction with both multiscopic imagery and stereoscopic imagery, allowing for use of conventional $3 \mathrm{D}$ programming as well as pre-rendered multi-perspective images.

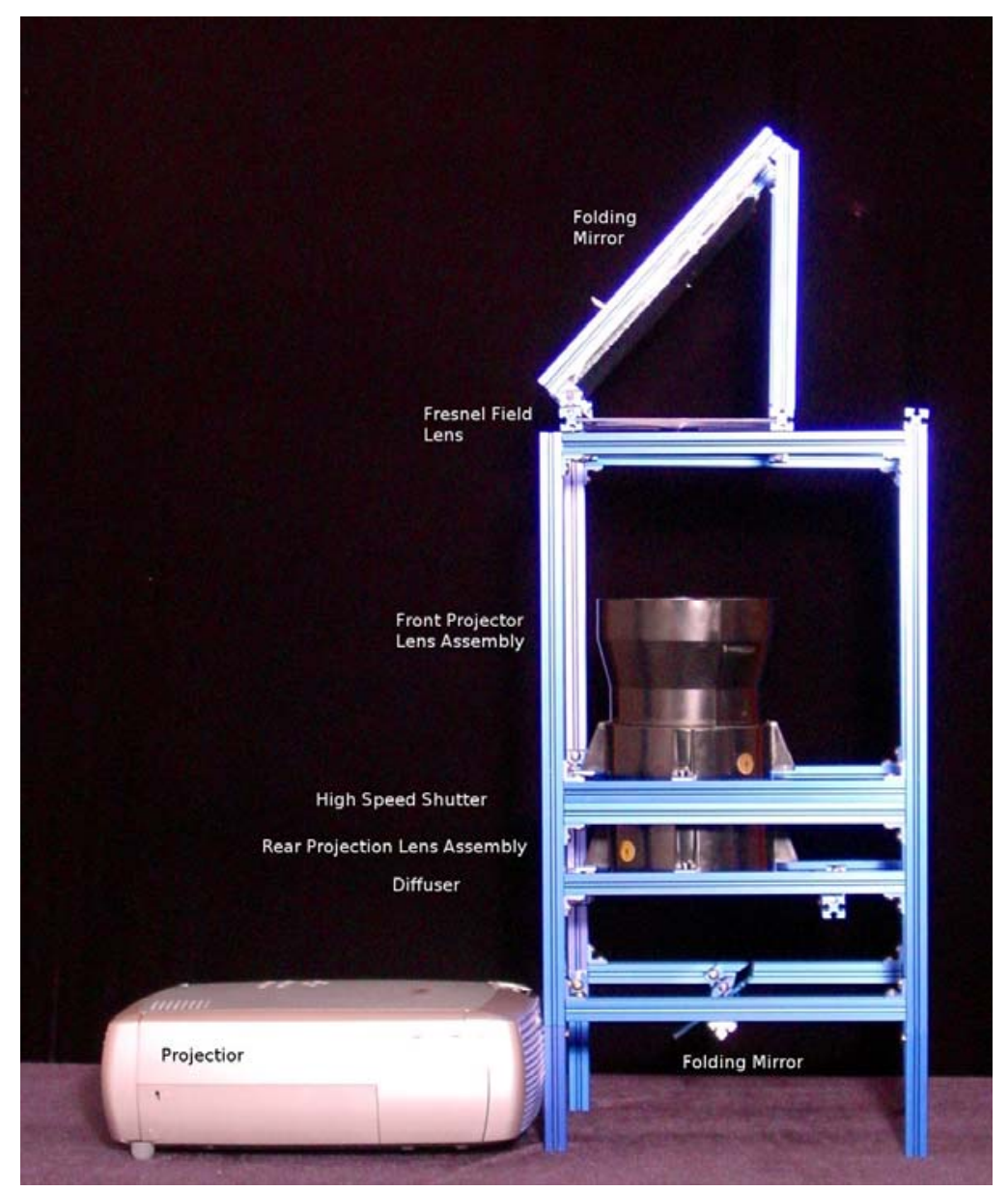

Figure 2. Initial Prototype of the Time Multiplex Autostereoscopic Display.

Design of the follow-on system is currently underway. The new system employs the use of a Texas Instruments DMD Discovery 3000 SLM to provide extremely rapid display of frames ( 480 fps) coupled with the use of either a digital visual interface-digital (DVI-D) or low voltage differential signaling (LVDS) coupled with the PCI-express (PCI-e) bus to achieve multiple gigabit per second link between one or more GPUs and the DMD buffer system. Given the high framerates required, the use of a combined color wheel and metal halide lamp which is commonly employed with conventional DMD projectors is not desirable due to the high speed of operation required for the color wheel as well the challenges associated with synchronizing the variations of rotation speed. As a result the integration of a LED based light engine is under development. LED Light engines[7] for DMD projectors have the advantage of very rapid switching times ( 100ns), highly saturated color gamuts and relatively high levels of illumination. The design parameters of the system assume a 20" diagonal screen with 8-16 viewing zones. The ultimate aim of the system is to integrate the display under development as module of a clustered display which employ each individual display as a 
"pie slice" of multiple displays which may be combined to form a synchronously networked display as shown in Figure 3. Such an arrangement allows for the use of cluster rendering and multiple optical trains to allow for broad fields of view well suited to urban visualization.

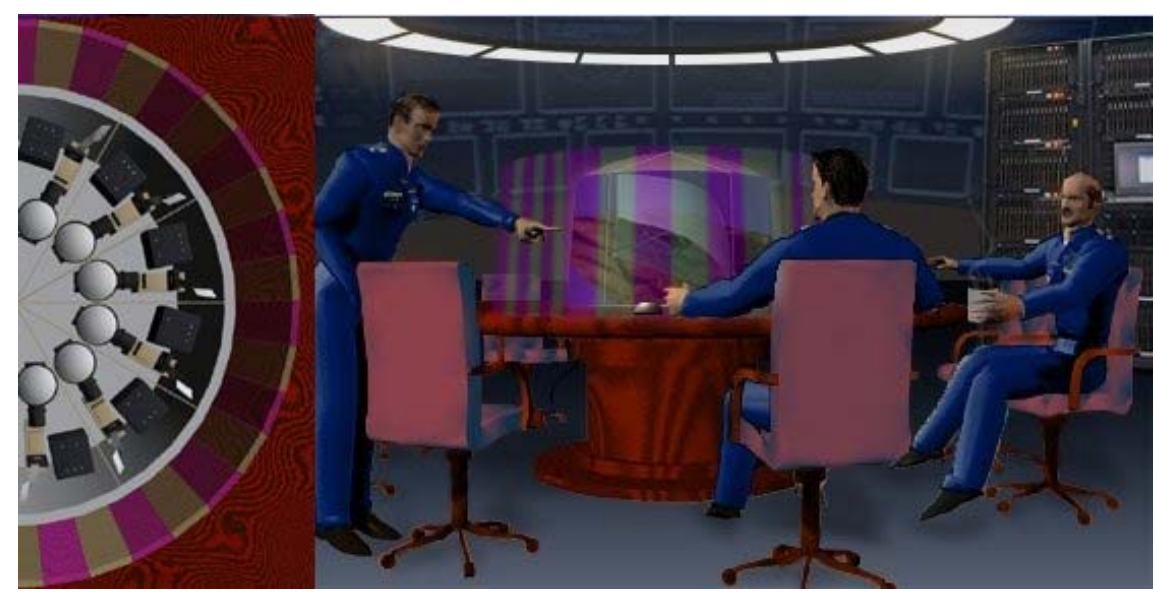

Figure 3. "Pie Slice” Display Cluster.

\subsection{Aspect From Point Display (AFPD)}

The AFPD consists of a time multiplexed display system which incorporates both conventional optics and a faceted holographic optical element (HOE) allowing for greater flexibility in the optical design. Two configurations are possible using this approach. The first integrates a series of holographic facets near the pupil of the system which image to the exit aperture in a manner similar to that of a conventional time multiplexed display. The second configuration consists of a series of facets near the image plane of the system, which selectively displays portions of separate images projected by the high-speed projector system. This latter approach is similar to that of integral display systems. Figure 4 illustrates this configuration. The AFPD is still in the early stages of development.

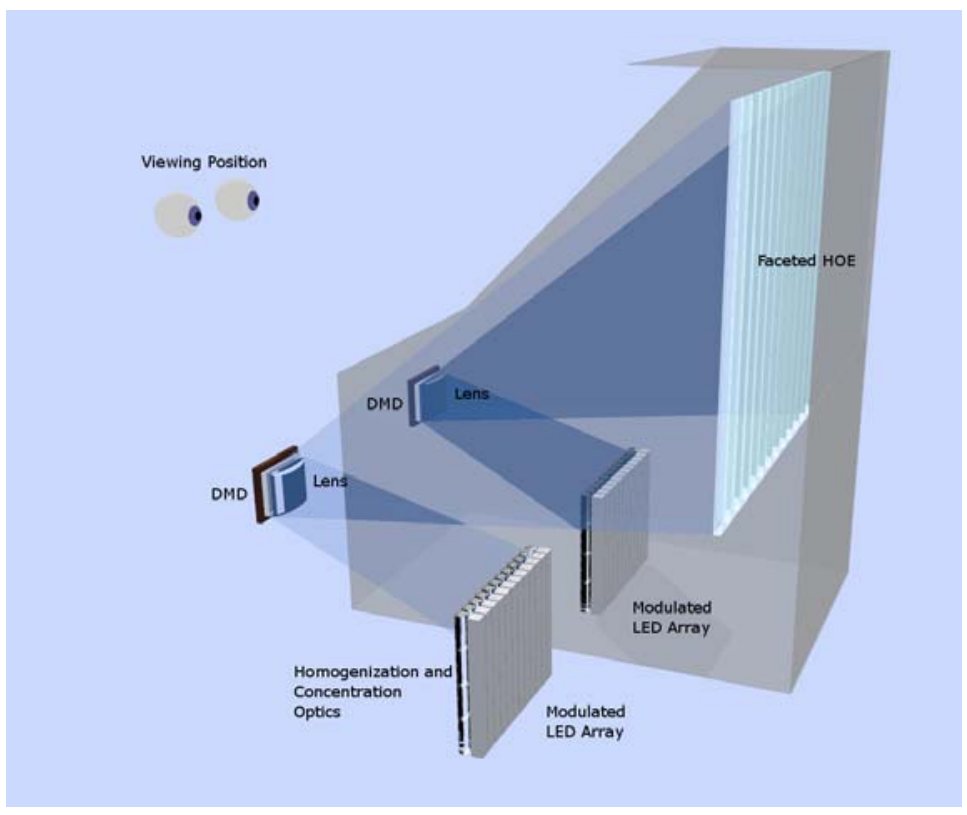

Figure 4. Aspect From Points Display Concept. 


\section{MODEL CAPTURE AND IMPORT}

In order to present timely and accurate models of urban landscapes it is necessary to be able to capture as well as import such 3D models. We have employed both methods to visualize urban landscapes, incorporating data collected by one of the authors $[8,9,10]$ as well as data derived from sources such as Google Earth.

\subsection{Model Capture}

The rapid and efficient capture of 3D models of urban areas is of great interest for several reasons. With increased storage capacity it is possible to store $3 \mathrm{~d}$ models of major urban areas for the purposes of navigation planning and visualization, as a result a variety of businesses most notably Google are pursuing the collection of such information. Given the sheer volume of data required for collection, rapid, largely automated methods will be required. In the case of military command and control as well as civilian response to disasters or terrorist attacks, the need exists to map new areas as well as obtain information on damage inflicted by battle or nature. In both cases, a means of rapidly capturing the 3D structure of an urban area is required. In addition it is equally essential to georeference these models in order to allow fusion with existing maps as well as position data generated by GPS tracking of vehicles and personnel.

The primary method of capture employed in our displays utilizes a combination of aerial photography and LIDAR scans to obtain the upper portions of buildings and structures as well as templates for registration of the models, coupled with ground level scans to obtain the facades of buildings and structures. The sequence of the algorithm is as follows:

- Aerial Photography and Registered Lidar scanning is performed at medium altitude. Point clouds of contiguous upper portions of buildings and structures are polygonized via Random Sample Consensus (RANSAC)-like algorithm forming the upper portion of the 3D model.

- The outlines of the lower portions of the model are formed by the outlines of the upper model.

- Vehicles equipped with registered cameras and two laser scanners then crisscross the streets of the area of interest. One laser scanner collects a vertical profile which is coupled with the registered camera to obtain the actual structure of the facades while a second horizontal laser scanner estimates the position of the vehicle based on the façade outline coupled with dead reckoning (it is assumed that GPS coverage is sporadic or distorted by clutter and multipath interference).

- The point cloud model is then registered to the façade outline via the use of a by means of Monte-CarloLocalization (MCL), a powerful Bayesian particle filtering algorithm. Finally the lower point cloud is surfaced to form a contiguous 3D model of the scene.

The resulting raw model is then translated into a scenegraph format (VRML97) incorporating multiple level of detail (LOD) models in order to allow for the precise control of image framerates. This is of particular importance in the case of time multiplexed display systems in that is necessary to render each frame for each of the perspectives within the refresh rate of the displays.

The Primary model used in out present work is shown in Figure 5 and consists of a model of downtown Berkley California generated by the method described above. The final model has been optimized for LOD control to optimize render times. This model has been successfully rendered for 3D visualization employing both stereoscopic and multiscopic techniques, which will be described in the following section. 


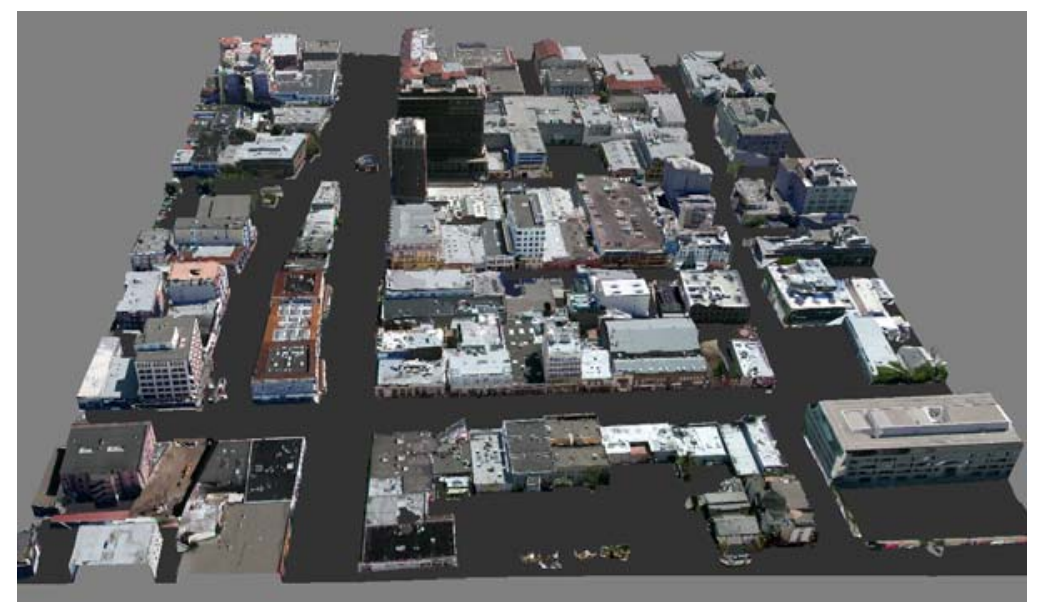

Figure 5. Scanned Model of Urban Area.

\subsection{Model Import}

While the highly detailed models obtained by direct scanning of urban areas provide an optimal source of models for specific areas of interest, it is often necessary to incorporate less detailed models covering a broader overall area. A good example of such a set of low detail models is the set of models employed in Google Earth for their 3D representations of large urban areas. Despite the lack of detail of these models they are often sufficient to assess important features of urban terrain such as lines of sight, lines of flight, and airflow. Thus the capacity to import this information is often essential. Given that a broad variety of differing formats and methods for access are employed for differing data sets. In order to avoid the complexity of such protocols our initial proof of concept approach employed intercepting the urban model at the level of the scene description language. In particular models were extracted by employing the OpenGL Extractor (OGLE) [11] package to capture the three dimensional scene by capture of the stream of incoming OpenGL commands. The sequence was then parsed, filtered and then reregistered to obtain a model compatible with our software an example of which is shown in Figure 6. Future refinements of this process will operate via direct access of the selected 3D databases.

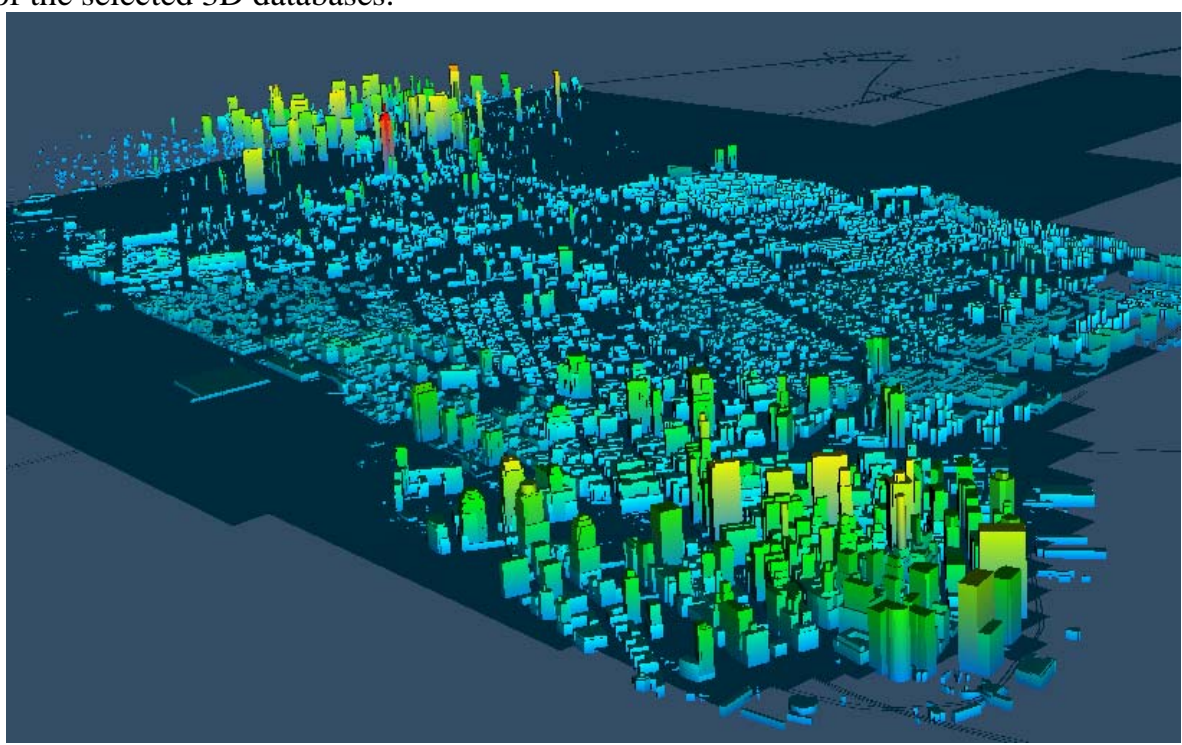

Figure 6. Image of Manhattan Island color coded by building height . (Source Data (C) 2006 Google, (C) 2006 Sandborn, (C) 2006 Europa Technologies) 


\section{SOFTWARE METHODS}

Visualization of multiscopic computer graphics for specific 3D display configurations imposes unique demands upon the rendering software employed. For each distinct viewing zone an image with the appropriate shear perspective matched to the geometry of the viewer and the display must be generated [12,13,14]. This rendering process must be performed at a high rate of speed to allow for smooth interaction with the model. In addition, given $\mathrm{n}$ views the rendering system must maintain $2 \mathrm{n}$ buffers to allow for proper display (a practice analogous to double buffering in monoscopic rendering and quad buffering in stereoscopic rendering). As a result it is generally necessary to extend the rendering engine employed to support these requirements.

Within our present implementation of the urban visualization system several rendering engines have been employed to achieve stereoscopic and multiscopic viewing of urban scenes. The first approach is to employ conventional stereoscopic rendering engines and alternate between left and right views. This results in a series of viewing positions that alternate between stereoscopic and pseudoscopic (i.e. inside out) imagery. These stereoscopic approaches may be achieved with integrated stereoscopic drivers such as the consumer stereo driver provided by nVidia. This allows for the direct use of many preexisting rendering engines and VRML browsers without appreciable modification. This has allowed for the display of our urban models via several different rendering engines, including in-house display programs as well as the Cortona VRML brouser developed by Parallel Graphics.

While the use of the stereoscopic display mode allows for simple compatibility with preexisting software, the presence of pseudoscopic viewing zones as well as lack of look around capabilities limits its utility. As a result it is necessary to develop true multiscopic display software. Initial development of multiscopic displays focused on the use of prerendered animations of display sequences in conjunction with synchronized playback displays. Within our initial display system the Guimigolus extension of the VisionEgg vision stimulation system ${ }^{15}$. This allows for stable display of multiple viewing zones (4-6) but is limited to the framerates of conventional displays ( $<140 \mathrm{fps}$ ).

In order to allow for the simultaneous display of 8-16 viewing zones we are presently developing a custom rendering engine which is optimized for rapid rendering of imagery with moderate complexity and dynamic range while allowing for direct transfer of the imagery to the DMD based SLM employed by our displays. In order to achieve this, our present focus is on the use of LOD control to strictly limit rendering time, the use of multiple GPU arrangements via the scalable link interface (SLI) architecture and the development of rapid transfer methods between the graphics buffer memory and the DMD buffer.

\section{CONCLUSION}

The visualization of dense urban areas is of increasing importance for military, and civilian applications. The complexity of such visualizations introduces ambiguities that are difficult to resolve within conventional displays. The use of true 3D displays which incorporate multiscopic "look around" and collaborative capabilities resolve many of these limitations. Within this paper we have described the development of multiscopic displays that have been used successfully to visualize models derived from direct scanning as well as preexisting models of urban areas.

\section{ACKNOWLEDGEMENTS}

This material is based upon work supported by the USAF/AFRL at Wright Patterson AFB under Contract No. FA865006-C-6617. Any opinions, findings and conclusions or recommendations expressed in this material are those of the author(s) and do not necessarily reflect the view of the USAF/AFRL, Wright Patterson AFB

\section{REFERENCES}

1 V. Markov, S. Kupiec and A. Travis, Recent advances in temporally multiplexed and point aspect autostereoscopic displays, Proc. SPIE, Volume 6225, pp. 622509 (2006).

2 V. Markov, S. Kupiec and A. Travis, Two approaches in the development of auto stereoscopic 3D display systems, 7th International Symposium on Display Holography, St Asaph, Wales, UK, (2006) 
3 Hopper, D.G., "Reality and Surreality of 3-D Displays: Holodeck and Beyond" Invited Keynote Paper in the Electronic Information Display Conference of Society for Information Display ( 'SID@EID' ), held at the ExCel Conference and Exhibition Centre, Central London, England UK ( Nov 21 - 23, 2000).

4 J.R. Moore, N. A. Dodgson, A.R.L. Travis, S.R. Lang, “Time-Multiplexed Color Autostereoscopic Display,” Proc. SPIE 2653, pp. 10-19, 1996.

5 Yu. N. Denisyuk, V.B. Markov, N.M.Ganzherli, "Projection of a Three Dimensional Image by the Method of Aspects Focused to Points," Optics and Spectroscopy, 84, pp 94-98, 1998

6 Yu. Denisyuk, V.Markov, N. Ganzherli, "Three-Dimensional image projection with aspects focused-in-spots display”, Proc. SPIE, 3011, pp. 2-7, 1997.

7 Keuper, M. H., Paolini, S., Harbers, G., Tsang, P., Ultra-Compact LED based Image Projector for Portable Applications, SID 03 DIGEST,pp 713-715, 2003.

8 C. Früh and A. Zakhor, "Constructing 3D City Models by Merging Ground-Based and Airborne Views" Computer Graphics and Applications, November/December 2003, pp. 52 - 61.

9 C. Früh, R. Sammon, and A. Zakhor, "Automated Texture Mapping of 3D City Models With Oblique Aerial Imagery" in 2nd International Symposium on 3D Data Processing, Visualization, and Transmission, Thessaloniki, Greece, September 2004

10 Ali Lakhia and A. Zakhor, "Interactive Rendering for Large City Models in 2nd International Symposium on 3D Data Processing, Visualization, and Transmission, Thessaloniki, Greece, September 2004”

11 http://ogle.eyebeamresearch.org/

12 Grinberg, V., Podnar, G., and Seigel, M., "Geometry of Binocular Imaging," in Proceedings of the SPIE, vol. 2177, pp. 56 - 65, 1994.

13 Grinberg, V. S., Podnar, G. W., and Siegel, M. W.. Geometry of binocular imaging II: The augmented eye. In Stereoscopic Displays and Applications VI, pages 142-9, 1995

14 Grinberg, V. S. and Siegel, M. W. Geometry of binocular imaging III: Wide-angle and fish-eye lenses. Stereoscopic Displays and Applications VII, (1996)

15 http://www.visionegg.org/ 